\title{
Perspective Application of the Circular Economy in the Blue Biotechnology: Microalgae as Sources of Health Promoting Compounds
}

\author{
Igor Fernandes ${ }^{* 1,2}$, Rafael Pinto ${ }^{1}$, Roberto Aguiar ${ }^{1}$ and Rogério Correia ${ }^{1,2}$ \\ ${ }^{1}$ Phytoalgae, São Martinho, Portugal \\ ${ }^{2}$ University of Madeira, Funchal, Portugal
} *Corresponding author: Igor Fernandes, Phytoalgae and University of Madeira,
Funchal, Portugal.
Received Date: June 08, 2020

Published Date: June 24, 2020

\section{Introduction}

Algae belong to a wide range of photosynthetic organisms that grow in aquatic environments (rivers, oceans and lakes) [13]. They are commonly classified into microalgae (unicellular and simple multicellular structures) and macroalgae (complex multicellular structures) depending on their size and cellular organization. Microalgae are microscopic single cells which may be prokaryotic (e.g. cyanobacteria) or eukaryotic (e.g. green algae) [13]. Furthermore, they are photoautotrophic microorganisms with the ability to use solar energy, reduce inorganic carbon to organic matter and producing biomass simultaneously [4].

Additionally, they are a good source of biochemical compounds, such as, polysaccharides, lipids, proteins, vitamins and pigments, which can be extracted through several conventional techniques (e.g. maceration, aqueous and Soxhlet extraction) [1,3,5]. Finally, the existence of natural products with strong antioxidant activity augments the economical and nutritional potential for food, pharmaceutical, and nutraceutical industry [1].

The trend in microalgal market is the production of biomass as a source of added value compounds [1,2]. The biomass can be used in several fields, like development of cosmetics for the treatment of skin disorders (e.g. aging and tanning), production of bioactive compounds in pharmaceuticals (e.g. vaccines that can be administered orally), production of low-cost natural-based biofertilizers and manufacture of biofuels (e.g. biodiesel, bioethanol and biohydrogen) [1,2]. Microalgae are crucial for human health as they are a source of polyunsaturated fatty acids (PUFA), which have shown to be effective in the prevention and treatment of several diseases [6]. PUFAs, especially omega 3 PUFAs ( $\alpha$-linolenic acid: C18:3n-3; EPA: C20:5n-3 and DHA: C22:6n-3), are widely used in the prevention and treatment of cancer, type 2 diabetes and several cardiovascular diseases [6].

\section{Agro-Industrial Residues}

Nowadays, there is a worldwide interest in the production of microalgae combined with agro-industrial residues, to reduced costs and promote circular economy, however this is an area that requires further development [7]. Additionally, the exhaustion of fossil fuels and the effects of global warming has increased the interest in renewable sources that could replace non-renewable energy (e.g. cropping residues, sugar beet bagasse, cardoon-waste, tomato-waste, spent coffee-grounds, [8] cheese whey and corn steep liquor [9]).

Agro-industrial residues consist of lignocellulose, a compact and partly crystalline structure, which is a combination of linear crystalline polysaccharides (e.g. cellulose), branched non-cellulosic and non-crystalline heteropolysaccharides (hemicelluloses) and branched lignin (non-crystalline) [10]. The agro-industrial residues can be used by several industrial fields since they are cheap, abundant and can provide environmental and economic benefits 
[10]. When discarded in the environment, these by-products release a large amount of nutrients (considered inorganic pollutants), such as, nitrogen and phosphorus [9]. These environmental disposal problems can be used as an alternative culture medium in the production of microalgae $[10,11]$. Furthermore, it is possible to obtain algal biomass with significant added value that could be used in the manufacture of several pharmaceuticals, cosmetics and nutritional supplements $[9,13]$. One of the limitations is that most microalgae cannot directly ingest and assimilate these residues, because of their complex composition (e.g. polysaccharides, proteins and fatty acids) [14]. Therefore, the transformation and modification of these agro-industrial residues may be required, which is fulfilled through physical (e.g. milling [10]and muffle furnace [15]) chemical (e.g. use of organic solvents [16]) and enzymatic processes $[13,17]$ resulting in easy to-assimilate hydrolysates [14].

Several studies have the purpose of determining the efficiency of agriculture and industry residues on the growth of different species of microalgae. For example, Chlorella vulgaris was cultured in cheese whey (CW, 10 gL-1 lactose), 1\% corn steep liquor (CC) and $2 \%$ vinasse $(\mathrm{CV})$ under autotrophic (CA) and mixotrophic conditions to determinate the biomass, protein and carbohydrates content [9]. C. vulgaris cultivated in a culture medium supplemented with $1 \%$ CC showed the maximum biomass concentration $(2.10 \mathrm{gL}-$ 1), whereas in a culture medium supplemented with $\mathrm{CW}$ vinasse exhibited a significant decrease in biomass $(1.60 \mathrm{gL}-1$ and 0.77 gL1 , respectively) [9].

C. vulgaris cultured with $\mathrm{CC}$ has achieved the highest protein content $(43.33 \%)$ whereas with the other by-products obtained a half-protein content (CW: 23.20\%; CV: 22.23\%) [9]. Conversely, C. vulgaris cultured with $\mathrm{CW}$ obtained a higher carbohydrates content (44.85\%) than CA and CV (39.34\% and 36.50\%, respectively) and a double the amount of carbohydrates compared to CC (25.41\%) [9].

Additionally, C. vulgaris was cultivated under photoautotrophic and mixotrophic conditions using a hydrolysed cheese whey powder solution to evaluate the maximum biomass production and the content of lipids, carotenoids and pigments [13]. Results shown that C. vulgaris cultivated under mixotrophic conditions presented biomass content with a 2.9-fold increase compared to photoautotrophic conditions (3.58 gL-1 and 1.22 gL-1, respectively) [13]. On the other hand, C. vulgaris cultivated under photoautotrophic conditions has given higher levels of lipids, pigments and carotenoids (42\%, $0.74 \%$ and $0.23 \%$, respectively) [13].

The potential of industrial cane molasses as a carbon source for the growth of Chlorella zofingiensis was studied under heterotrophic conditions to determinate the lipids, ketocarotenoid astaxanthin content, and fatty acid profile [18]. According to the results, C. zofingiensis cultured with different concentrations of pre-treated molasses achieved a biomass, lipid and astaxanthin productivities of 1.55 gL-1 day-1, 0.71 gL-1 day-1 and $1.7 \mathrm{mgL}-1$ day-1, respectively [18]. Besides the fatty acid profile of C. zofingiensis has higher levels of polyunsaturated fatty acids then monounsaturated fatty acids and the highest percentage of polyunsaturated fatty acids (49.16\%) was obtain with the concentration of 5 gL-1 sugar [18]. Furthermore, palmitic acid, hexa-decadienoic acid, oleic acid, linoleic acid and $\alpha$-linolenic were the major fatty acids presented in C. zofingiensis profile and represented more than $85 \%$ of total fatty acids [18].

The efficiency of two organic carbon sources, residual corn crop hydrolysate and corn silage juice on the growth, were studied (biomass and lipids content and fatty acids profile) under heterotrophic, photoautotrophic and mixotrophic conditions in a bacteria-Chlorella sp. Consortium [14]. Results shown that maximum microalgal biomass (0.8 gL-1) was obtained with 1 gL-1 of residual corn crop hydrolysate whatever the trophic conditions [14]. Under mixotrophic conditions, the use of residual corn crop hydrolysate led to an increase of 21 and $22 \%$ in the biomass produced in comparison to silage juice [14]. Additionally, this increase varied between 11 and 28\% under heterotrophic conditions [14]

Chlorella sp. cultured under photoautotrophic conditions accumulated almost $30 \%$ of total lipids, which was higher than under heterotrophic and mixotrophic conditions (7\% and 15\%, respectively) [14]. According to the fatty acid profile, Chlorella sp. presented the highest concentration of fatty acids under mixotrophic conditions in residual corn crop hydrolysate (15.5 mg g-1 dry weight) whereas the lowest was under heterotrophic conditions with silage juice ( $0.21 \mathrm{mg}$ g-1 dry weight) [14]. Under photoautotrophic conditions, the major classes of lipids in Chlorella sp. fatty acid profile were palmitic, gamma-linolenic and $\alpha$-linolenic fatty acids, which represented almost $90 \%$ of the total fatty acids measured [14]. In mixotrophic and heterotrophic cultures the relative composition of the major fatty acids diversified, as palmitoleic, stearic and oleic acids were not detected [14].

In another study, Chlorella vulgaris and Scenedesmus quadricauda were grown in absence and presence of different humic-like substances extracted from agro-industrial wastes (digestate from the waste of an agro-livestock farm (D-HL), oil extraction residues from rape B-HL, Brassica napus and tomato residues (T-HL)) to evaluate their biomass, lipids, carbohydrates, chlorophylls content and fatty acid profile [14]. The cultivation of C. vulgaris and S. quadricauda in a culture medium supplemented with 100 mgL- 1 of D-HL led to an increase in the biomass compared to BG11 (control) culture medium with $41 \%$ and $31 \%$ for C.vulgaris and S. quadricauda, respectively [16]. On the other hand, the cultivation in $100 \mathrm{mg} \mathrm{L}-1$ of T-HL produced $29 \%$ and $21 \%$ fold increase for C. vulgaris and S. quadricauda and all other cases the biomass production was not significantly different [16]. The total lipid content of both species increased in the presence of HLs under 
all experimental conditions [16]. Besides that, C. vulgaris cultured with B-HL (both concentrations) and D-HL2 presented a $10 \%$ decrease in comparison with the control [16].

In S. quadricauda, the presence of the HLs induced an increase of the lipid content, with significant higher values than observed for the control [16]. Furthermore, for both species, B-HL (both doses) led to an increase of the total carbohydrate content compared with the control [16]. Regarding the monosaccharides, in C. vulgaris the treatment with B-HL1 and B-HL2 induced an increase in the glucose content (19.18\% and $17.79 \%$, respectively) compared with control (15.27\%).and a large increase in galactose content [16].

Moreover, the treatment with B-HLs led to a similar behaviour at both concentrations regarding glucose, whereas an increase in galactose and xylose was obtained only at the lowest and highest concentrations, respectively [16]. Furthermore, B-HLs led to an increase in both chlorophylls a and b in both species, in comparison with the control [16]. Contrarily, treatment with D-HLs and T-HLs induced, in general, a decrease in the total carbohydrates and chlorophylls content for both microalgae [16].

Regarding C. vulgaris cultivated in BG11, the fatty acid profile contained mainly palmitic (34.5\%), oleic $(11.7 \%)$, stearic $(7.6 \%)$, linoleic (5.87\%), elaidic (5.47\%) and alpha-linoleic (4.41\%) acids [15]. The treatment with D-HLs and T-HLs led to a reduction in the palmitic, stearic and oleic acids and simultaneously increased the quantity of linoleic and alpha-linoleic acids [16]. On the other hand, treatment with B-HLs induced an increase in linoleic acid content and a decrease in palmitic and stearic acids [16].

With respect to S. quadricauda cultivated in BG11, it contained mainly palmitic (23.44\%), oleic (12.42\%), alpha-linoleic (10.86\%) and linoleic (8.85\%) acids [16]. In S. quadricauda, the treatment with D-HLs increased the unsaturated fatty acids (gamma-linolenic, alpha-linolenic and linolenic acids) relatively with the control [16]. Moreover, with the addition of B-HL1 it was observed an increase of the saturated fatty acids, particularly palmitic (24.01\%), behenic (2.41\%), myristic (1.93\%) and stearic (1.37\%) acids [16].

\section{Conclusion}

Nowadays there is an increasing demand to produce microalgae due to the production of high value compounds. Furthermore, these microorganisms can assimilate, in some extend, inorganic pollutants that are produced by agronomical industry and therefore promote a circular economy. Agro-industrial residues are considered one of the largest sources of wastes in the world, which could release a large amount of inorganic pollutants that promote the deterioration of the environment and the loss of potentially valuable materials. These residues are constituted by several minerals, such as, magnesium, iron and potassium which are important to human health.

The production of microalgae using agro-industrial wastes can be considered as a feasible strategy to mitigate the environmental problems caused by the disposal of these residues and decreasing the production costs of microalgae biomass. Furthermore, agroindustrial residues are widely abundant and inexpensive, making them an ideal material to increase the nutritional potential of microalgae for the food, pharmaceutical and nutraceutical industries.

\section{Acknowledgment}

This study was supported by project M1420-01-0247FEDER-000023 of Instituto de Desenvolvimento Empresarial da Madeira (IDE, IP-RAM).

\section{Conflict of Interest}

No Conflict of Interest.

\section{References}

1. Khan M, Shin J, Kim J (2018) The promising future of microalgae: current status, challenges, and optimization of a sustainable and renewable industry for biofuels, feed, and other products. Microb Cell Fact 17(1): $1-21$.

2. Bhalamurugan G, Valerie O, Mark L (2018) Valuable bioproducts obtained from microalgal biomass and their commercial applications: A review. Environ Eng Res 23(3): 229-241.

3. Barkia I, Saari N, Manning S (2019) Microalgae for High-Value Products Towards Human Health and Nutrition. Mar Drugs 17(5): 304.

4. Fernández F, Gómez-Serrano C, Fernández Sevilla J (2018) Recovery of Nutrients from Wastewaters Using Microalgae. Front Sustain Food Syst 2(59): 1-13.

5. Tang G, Suter P (2011) Vitamin A, Nutrition, and Health Values of Algae: Spirulina, Chlorella and Dunaliella. J Pharm Nut Scie 1: 111-118.

6. Priyadarshani I, Rath B (2012) Commercial and industrial applications of micro algae-A review. J Algal Biomass Utln 3: 89-100.

7. León Vaz A, León R, Díaz Santos E, Vigara J Raposo S (2019) Using agroindustrial wastes for mixotrophic growth and lipids production by the green microalga Chlorella sorokiniana. New Biotechnology 51: 31-38.

8. De Corato U, De Bari I, Viola E, Pugliese M (2018) Assessing the main opportunities of integrated biorefining from agrobioenergy co/byproducts and agroindustrial residues into high-value added products associated to some emerging markets: A review. Renewable and Sustainable Energy Reviews 88: 326-346.

9. De Melo R, de Andrade A, Bezerra R, Correia D, de Souza V, et al. (2018) Chlorella vulgaris mixotrophic growth enhanced biomass productivity and reduced toxicity from agro-industrial by-products. Chemosphere 204: 344-350.

10. Nigam P, Gupta N, Anthwal A (2009) Pre-treatment of Agro-Industrial Residues. Biotechnol Agr Ind Res 2: 13-33.

11. Calixto C, Santana J, de Lira E, Sassi P, Rosenhaim R, et al. (2016) Biochemical compositions and fatty acid profiles in four species of microalgae cultivated on household sewage and agro-industrial residues. Bioresour Technol 221: 438-446.

12. Mirzaie M, Kalbasi M, Mousavi S, Ghobadian B (2015) Statistical Evaluation and Modeling of Cheap Substrate-Based Cultivation Medium of Chlorella vulgaris to Enhance Microalgae Lipid as New Potential Feedstock for Biolubricant. Prep Biochem Biotechnol 46(4): 368-375.

13. Abreu A, Fernandes B, Vicente A, Teixeira J, Dragone G (2012) Mixotrophic cultivation of Chlorella vulgaris using industrial dairy waste as organic carbon source 118: 61-66.

14. Gélinas M, Pham T, Boëns B, Adjallé K, Barnabé S (2015) Residual corn crop hydrolysate and silage juice as alternative carbon sources in microalgae production. Alg Res 12: 33-42. 
15. Miranda T, Arranz J, Montero I, Román S, Rojas C, et al. (2012) Characterization and combustion of olive pomace and forest residue pellets. Fuel Processing Technology 103: 91-96.

16. Puglisi I, Barone V, Sidella S, Coppa M, Broccanello C, et al. (2018) Biostimulant activity of humic-like substances from agro-industrial waste on Chlorella vulgaris and Scenedesmus quadricauda. Europ J Phyc 1: $1-10$.
17. Cheng Y, Zhou W, Gao C, Lan K, Gao Y, et al. (2008) Biodiesel production from Jerusalem artichoke (Helianthus Tuberosus L.) tuber by heterotrophic microalgae Chlorella protothecoides. J Chem Technol Biotechnol 84(5): 777-781.

18. Liu J, Huang J, Jiang Y, Chen F (2012) Molasses-based growth and production of oil and astaxanthin by Chlorella zofingiensis. Bior Technol 107: 393-398. 